\title{
miR-204-5p promotes diabetic retinopathy development via downregulation of microtubule-associated protein 1 light chain 3
}

\author{
XIN-BANG MAO ${ }^{1}$, YAN-HUA CHENG ${ }^{1}$ and YAN-YING XU ${ }^{2}$ \\ ${ }^{1}$ Department of Ophthalmology, The Second Affiliated Hospital of Nanchang University, Nanchang, Jiangxi 330006; \\ ${ }^{2}$ Department of Ophthalmology, Sir Run Run Shaw Hospital, Zhejiang University School of Medicine, \\ Hangzhou, Zhejiang 310016, P.R. China
}

Received October 20, 2017; Accepted December 12, 2018

DOI: $10.3892 /$ etm.2019.7327

\begin{abstract}
Diabetic retinopathy (DR) is a chronic and progressive complication of diabetes mellitus. DR impairs sight due to neuronal and vascular dysfunction in the retina. It is critical to investigate the pathogenesis of DR to develop effective treatment. In the present study, a streptozotocin (STZ)-induced diabetic rat model was constructed and the expression of microRNA (miR)-204-5p and vascular endothelial growth factor (VEGF) were determined. Immunohistochemistry, reverse transcription-quantitative polymerase chain reaction (RT-qPCR) and western blotting were employed to detect the effects of miR-204-5p on the expression of microtubule-associated protein 1 light chain 3 (LC3B). RT-qPCR analysis demonstrated that miR-204-5p and VEGF were significantly upregulated in the retina tissue of diabetic rats compared with the control group $(\mathrm{P}<0.01)$. Immunohistochemistry and western blotting revealed that the protein expression levels of LC3B-II and the ratio of LC3B-II/LC3B-I were significantly suppressed in the diabetes group compared with the control $(\mathrm{P}<0.01)$. In retinal tissues, anti-miR-204-5p treatment significantly enhanced the protein expression levels of LC3B-II and the ratio of LC3B-II/LC3B-I and these levels were significantly reduced in response to miR-204-5p mimic treatment compared with the negative miR control $(\mathrm{P}<0.01)$. In rat retinal endothelial cells isolated from diabetic rats, anti-miR-204-5p treatment increased the number of autophagic vacuoles, and significantly promoted LC3B-II expression and the LC3B-II/LC3B-I ratio
\end{abstract}

Correspondence to: Professor Yan-Ying $\mathrm{Xu}$, Department of Ophthalmology, Sir Run Run Shaw Hospital, Zhejiang University School of Medicine, 3 East Qingchun Road, Hangzhou, Zhejiang 310016, P.R. China

E-mail: xyy25801@163.com

Abbreviations: DR, diabetic retinopathy; STZ, streptozotocin; LC3B, microtubule-associated protein 1 light chain 3; VEGF, vascular endothelial growth factor

Key words: diabetic retinopathy, autophagy, microRNA-204-5p, microtubule-associated protein 1 light chain 3 compared with the negative control $(\mathrm{P}<0.01)$. The results of the present study revealed that miR-204-5p downregulated the expression of LC3B-II to inhibit autophagy in DR. Therefore, miR-204-5p may be considered as a novel effective therapeutic target during the development of DR.

\section{Introduction}

Diabetes mellitus is one of the most common types of chronic disease with extensive morbidity and mortality worldwide (1). Diabetic retinopathy (DR) is the most common microvascular complication of diabetes and is a frequent cause of preventable blindness worldwide (2). In 2010, 3.7 million people were visually impaired and 0.8 million were blind due to DR (3). Various metabolic disorders have been associated with the onset of DR (4); however, the connection between metabolic abnormalities and the development of $\mathrm{DR}$ requires further investigation. At present, the molecular mechanisms underlying the pathogenesis of DR remain unknown and there are no effective treatments or preventative approaches for DR. The present study aimed to investigate risk factors for developing DR and determine a novel target for the treatment of this particular complication.

Autophagy is a conserved metabolic process that is characterized by the degradation and recycling of dysfunctional proteins or organelles (5). In the process of autophagy, the autophagosome is formed for the isolation of targeted or non-specific materials (6); microtubule-associated protein 1 light chain 3 (LC3B), comprising two forms (LC3B-I and LC3B-II), is essential for the formation of the autophagosome (7). Upon the induction of autophagy, LC3B-I is converted to LC3B-II, which integrates into the membrane of the autophagosome (8). Studies have demonstrated that autophagy is one of the major causative factors involved in the pathogenesis of DR $(9,10)$. LC3B-II has been associated with the extent of autophagosome formation (11) and serves as a valuable molecular biomarker for the detection of autophagic activity (12); thus, LC3B-II may be an effective therapeutic target for the treatment of DR.

MicroRNAs (miRNAs/miRs) are small non-coding RNAs, which modulate the expression of target mRNAs via the post-transcriptional inhibition of translation (13). It has been revealed that miRNAs may be directly or indirectly 
involved in several diseases by regulating the expression of numerous genes (14). miR-125b-5p has been associated with the progression of DR by regulating the expression of specificity protein 1 (15). Furthermore, miRNA-21 was reported to negatively regulate the expression of peroxisome proliferator-activated receptor- $\alpha$ in the retina of mice with diabetes (16) and modulate the expression of prorenin receptor-induced vascular endothelial growth factor (VEGF) under hyperglycemic conditions (17). miR-200b was reported to be involved in the pathogenesis of DR in an VEGF-independent manner (18). These findings indicate the considerable potential of miRNAs for therapeutic application in the treatment of DR. Previous studies reported several differentially expressed miRNAs in the development of early stage DR by using a miRNA microarray analysis $(15,19)$, including miR-135b-5p, miR-145-5p, miR-146a-5p, miR-199a-5p and miR-204. It has also been reported that miR-204-5p was involved in diabetic keratopathy (20). However, the role of miR-204-5p in DR remains elusive. Reverse transcription-quantitative polymerase chain reaction (RT-qPCR) revealed that miR-204-5p and VEGF were upregulated in the retina of rats with streptozotocin (STZ)-induced diabetes, whereas the protein expression levels of LC3B-II and the ratio of LC3B-II/LC3B-I were significantly decreased. Anti-miR-204-5p treatment promoted the expression of LC3B-II and the ratio of LC3B-II/LC3B-I; however, these levels were suppressed in response to exposure to miR-204-5p mimic. The results of the present study suggested that miR-204-5p may be involved in the progression of DR by negatively modulating the expression of LC3B-II. These findings also indicated that modulation of retinal miR-204-5p expression may be considered as potential therapeutic strategy for the treatment of DR.

\section{Materials and methods}

Animals and grouping. A total of 60 male Sprague Dawley rats (aged 6-8 weeks; weighing, 180-220 g) were purchased from Laboratory Animal Services Centre of Hunan Slack Jingda Experimental Animal Co., Ltd. (Changsha, China). Rats were housed at $18-22^{\circ} \mathrm{C}$ with 12 -h light/dark cycles and access to standard food and water ad libitum. All procedures were approved by the Animal Ethics Committee of Second Affiliated Hospital of Nanchang University (Nanchang, China). Rats were fed with a high glucose, high fat diet (60\% standard chow, $10 \%$ lard, $10 \%$ egg yolk powder and 20\% sucrose). Following 8 weeks, rats were divided into two groups: The diabetic group $(n=50)$ and the control group $(\mathrm{n}=10)$. Rats in the diabetes group were injected with STZ (Sigma-Aldrich; Merck KGaA, Darmstadt, Germany) at $40 \mathrm{mg} / \mathrm{kg}$. At $72 \mathrm{~h}$ following STZ treatment, rats were considered diabetic providing their fasting blood glucose levels were $>16.7 \mathrm{mM}(21)$. The control group received $2 \mathrm{ml}$ of $0.1 \mathrm{M}$ sodium citrate buffer.

Anti-miR-204-5p [adenovirus AAV-U6-rno-miR-204-5 p-CAG-enhanced green fluorescent protein (EGFP); cat. no. MICA2014002038], miR-204-5p mimic (adenovirus AAV-U6 -mimic-rno-miR-204-5p-CAG-EGFP, 5'-UUCCCUUUGUCA UCCUAUGCCU-3') and negative control miRNA (neg-miR, adenovirus AAV-U6-mimic-rno-negative-miR-CAG-EGFP, 5'-UUCUCCGAACGUGUCACGUTT-3') were obtained from Shanghai GeneChem Co., Ltd. (Shanghai, China).
Diabetic rats were randomly divided into three groups: Neg-miR, anti-miR-204-5p and mimic groups. Diabetic rats in the anti-miR-204-5p group $(n=15)$ were injected with anti-miR-204-5p adenovirus $\left(4.1 \times 10^{12}\right.$ viral genome $\left./ \mathrm{ml}\right)$ via the caudal vein. Diabetic rats in the mimic group $(n=15)$ received miR-204-5p mimic adenovirus (4.1 $\times 10^{12}$ viral genome $\left./ \mathrm{ml}\right)$. The neg-miR group $(n=15)$ were injected with the same quantity of neg-miR adenovirus. At 8 weeks, retinal tissues were extracted for further investigation as detailed below.

Rats retinal epithelial cells (rRECS) culture. Five diabetic rats were anesthetized by intraperitoneal injection of pentobarbital sodium $(30 \mathrm{mg} / \mathrm{kg})$. Under anesthesia, the eyeball of diabetic rats was isolated from diabetic rats and following rats were sacrificed using a lethal dose of pentobarbital $(100 \mathrm{mg} / \mathrm{kg}$ body weight). The retina was separated from the eyeball and washed with Hanks balanced salt solution (H1020; Beijing Solarbio Science \& Technology Co., Ltd., Beijing, China) and minced into small pieces $(1 \mathrm{x} 1 \mathrm{~mm})$ with surgical scissors. Following filtration through a $30-\mu \mathrm{m}$ nylon sieve, the retina was digested with $2.5 \%$ trypsin for $30 \mathrm{~min}$ at $37^{\circ} \mathrm{C}$. Following centrifugation $(1,500 \times \mathrm{g}$ for $5 \mathrm{~min})$ at room temperature, rRECs were isolated and cultured in Dulbecco's modified Eagle's medium (DMEM; Gibco; Thermo Fisher Scientific, Inc., Waltham, MA, USA) containing $20 \%$ fetal bovine serum (FBS; Sigma-Aldrich; Merck $\mathrm{KGaA}$ ) at $37^{\circ} \mathrm{C}$ with $5 \% \mathrm{CO}_{2}$. Medium was replaced every three days. rRECs were identified for endothelial homogeneity by testing for immunoreactivity to factor VII antigen. rRECs were fixed with $4 \%$ paraformaldehyde, blocked with $2 \%$ BSA for $1 \mathrm{~h}$ at room temperature and incubated with mouse anti-factor VII antibody (cat. no. ab97614; Abcam, Cambridge, UK) at $4^{\circ} \mathrm{C}$ overnight. The cells were washed and incubated with Alexa Fluor ${ }^{\circledR}$ 647-conjugated donkey anti-rabbit immunoglobulin $\mathrm{G}$ secondary antibodies (cat. no. ab150075; Abcam) at $37^{\circ} \mathrm{C}$ for $2 \mathrm{~h}$. Then nuclear was stained with DAPI (Sigma-Aldrich; Merck KGaA) for 10 min at room temperature. Signals were detected with a fluorescent inverted microscope at a magnification of $x 200$. The third passage was prepared for subsequent experiments.

rRECs were seeded in 6-well plates at $1 \times 10^{6}$ cells/well and cultured in DMEM supplemented with 10\% FBS. At 70-80\% density, cells were transfected with $100 \mathrm{nM}$ miR-204-5p mimic (the mimic group), miR-204-5p inhibitor (the anti-miR-204-5p group) or neg-miR (the neg-miR group) with Lipofectamine 3000 (Roche Diagnostics, Basel, Switzerland). The transfection efficiency was determined by RT-qPCR. At $48 \mathrm{~h}$, cells were collected for follow-up experiments.

Transmission electron microscopy. rRECs at a density of $1 \times 10^{5}$ cells/well were fixed with $2.5 \%$ glutaraldehyde in $0.1 \mathrm{M}$ phosphate buffer $\mathrm{pH} 7.4$ for $2 \mathrm{~h}$ at room temperature. Following washing with $0.1 \mathrm{M}$ phosphate buffer, samples were stained with $1 \%$ osmium tetroxide for $1 \mathrm{~h}$ at room temperature. Following dehydration with an ethanol gradient, samples were embedded in LX-112 resin and heating at $70^{\circ} \mathrm{C}$ overnight. Sections were sliced to ultrathin sections $(70 \mathrm{~nm})$ and imaged with an FEI Tecnai Spirit transmission electron microscope (FEI; Thermo Fisher Scientific, Inc.) at magnifications of $\mathrm{x} 1,700$ and $\mathrm{x} 5,000$ 
RT-qPCR analysis. Total RNA was isolated from retinal tissues and rRECs using TRIzol reagent (cat. no. CW0580S; Beijing CWBio, Beijing, China) according to the manufacturer's protocol. RNA quality was evaluated by agarose electrophoresis and the quantity was determined via spectrophotometry at 260 and $280 \mathrm{~nm}$. A total of $500 \mathrm{ng}$ RNA was reverse transcribed into cDNA using the miRScript system (GenScript Co., Ltd., Nanjing, China) according to the manufacturer's protocols. qPCR was performed using SYBR Green qPCR Super mix (cat. no. CW0957M; Beijing CWBio) on a CFX96 Touch PCR Detection system (Bio-Rad Laboratories, Inc., Hercules, CA, USA). The thermocycling conditions were as follows: $95^{\circ} \mathrm{C}$ for 5 min followed by 40 cycles of $95^{\circ} \mathrm{C}$ for $30 \mathrm{sec}, 60^{\circ} \mathrm{C}$ for $30 \mathrm{sec}$. All experiments were performed with triplicate. The relative expression of VEGF was normalized to $\beta$-actin using the $2^{-\Delta \Delta \mathrm{Cq}}$ method (22). U6 was used as an endogenous control to analyze the expression of miR-204-5p. The primers for RT-qPCR were as follows: VEGF, forward, 5'-GAGTTAAACGAACGTACTTGCAGA-3' and reverse, 5'-TCTAGTTCCCGAAACCCTGA-3'; $\beta$-actin, forward, 5'-TGGCTGGGGTGTTGAAGGTC-3' and reverse, 5'-ATG GTGGGTATGGGTCAGAAGG-3'; miR-204-5p, forward, 5'-ACACTCCAGCTGGGTTCCCTTTGTCATCCTAT-3' and reverse, 5'-CTCAACTGGTGTCGTGGA-3'; and U6, forward, 5'-CTCGCTTCGGCAGCACA-3' and reverse, 5'-AACGCT TCACGAATTTGCGT-3'.

Western blotting. Proteins were isolated from retinal tissues and rRECs using radioimmunoprecipitation assay buffer (Sigma-Aldrich; Thermo Fisher Scientific, Inc.). Protein concentration was determined by bicinchoninic acid kit (Beyotime Institute of Biotechnology, Shanghai, China) according to the manufacturer's protocol. Equal amounts of protein $(60 \mu \mathrm{g})$ were separated using SDS-PAGE on $12 \%$ gels and transferred to polyvinylidene difluoride membranes (Millipore; Merck KGaA). Following blocking with 5\% non-fat milk for $1 \mathrm{~h}$ at room temperature, membranes were probed with rabbit anti-LC3B antibody (cat. no. ab51520; 1:3,000; Abcam) or anti- $\beta$-actin (cat. no. ab8226; $1: 1,000$; Abcam) overnight at $4^{\circ} \mathrm{C}$. Then, membranes were washed with TBS plus Tween-20 $(0.1 \%)$ and incubated with horseradish peroxidase-conjugated goat anti-rabbit secondary antibodies (cat. no. sc-516078; 1:1,000; Santa Cruz Biotechnology, Inc., Dallas, TX, USA) for $2 \mathrm{~h}$ at $37^{\circ} \mathrm{C}$. Target bands were developed with an enhanced chemiluminescence detection kit (Amersham; GE Healthcare, Chicago, IL, USA). Band densities were calculated using Quantity One software version 4.5.1 (Bio-Rad Laboratories, Inc.) and normalized to $\beta$-actin.

Immunohistochemistry. Retinal tissues were isolated from the different groups at 8 weeks following STZ injection, fixed with $10 \%$ freshly prepared ice-cold paraformaldehyde at $4{ }^{\circ} \mathrm{C}$ overnight and cut into $5-\mu \mathrm{m}$ sections. Sections were incubated with $5 \%$ bovine serum albumin (BSA; Beijing Solarbio Science \& Technology Co., Ltd.) at $37^{\circ} \mathrm{C}$ for $1 \mathrm{~h}$ to block the nonspecific sites. Then, sections were incubated with rabbit polyclonal anti-LC3B antibody $(1: 1,000)$ for $2 \mathrm{~h}$ at room temperature. Following washing with PBS (3x), slides were probed with horseradish peroxidase-conjugated goat anti-rabbit antibodies for $2 \mathrm{~h}$ at room temperature.
Immunoperoxidase binding was visualized by reaction with diaminobenzidine- $\mathrm{H}_{2} \mathrm{O}_{2}$ solution. Five non-overlapping fields were randomly captured by an inverted microscope at a magnification of $x 400$. LC3B-positive cells were stained in brown or dark yellow.

Statistical analysis. Data are presented as the mean \pm standard deviation. All statistical analysis was performed using SPSS 18.0 software (SPSS, Inc., Chicago, IL, USA). Statistical analyses were performed using one-way analysis of variance followed by Dunnett's post hoc tests. $\mathrm{P}<0.05$ was considered to indicate a statistically significant difference.

\section{Results}

miR-204-5p is overexpressed in diabetic rats. In the present study, a diabetic rat model was generated by intraperitoneal injection of STZ; the expression of miR-204-5p in the diabetic rat model was then investigated. To determine whether the diabetic rat model was successfully constructed, glucose levels were analyzed at day 3 following STZ administration. Glucose levels in the STZ-treated group $(23.01 \pm 3.71 \mathrm{mM})$ were significantly increased compared with the control group $(4.62 \pm 1.51 \mathrm{mM} ; \mathrm{P}<0.01$; data not shown). This suggested that a diabetic rat model was successfully constructed. As presented in Fig. 1A, compared with the control group, relative miR-204-5p expression levels in retina tissues of the diabetes group were significantly upregulated $(\mathrm{P}<0.01)$. In addition, expression VEGF mRNA levels in the diabetes group were significantly increased compared with the control group $(\mathrm{P}<0.01$; Fig. 1B). The results indicated that miR-204-5p and VEGF may be associated with the diabetes and contribute to the progression of DR.

LC3B-II expression and LC3B-II/LC3B-I are significantly suppressed in diabetic rats. LC3B-II levels are proportional to the number of autophagic vacuoles (7). To investigate the role of $\mathrm{LC} 3 \mathrm{~B}$ in the retina of diabetic rats, LC3B protein expression was determined via immunohistochemistry. As presented in Fig. 2A, compared with the control group, LC3B expression in the diabetic group was markedly reduced. These results revealed that the expression of LC3B was inhibited in retina tissues of diabetic rats.

Additionally, protein expression levels of LC3B were determined by western blotting. Compared with the control group, expression levels of LC3B-II in the diabetic group were significantly reduced ( $\mathrm{P}<0.01$; Fig. $2 \mathrm{~B}$ and $\mathrm{C})$. Furthermore, the ratio of LC3B-II/LC3B-I was significantly decreased in the diabetic group compared with the control ( $\mathrm{P}<0.01$; Fig. 2D). These data suggested that the expression of LC3B-II and the ratio of LC3B-II/LC3B-I may be suppressed during the development of DR.

miR-204-5p negatively regulates the expression of LC3B-II. To further investigate the mechanism by which miR-204-5p affects the progression of DR, anti-miR-204-5p and miR-204-5p mimic were administered to rats of the diabetes group. As presented in Fig. 3A, compared with the neg-miR group, anti-miR-204-5p treatment significantly suppressed miR-204-5p expression and miR-204-5p mimic treatment 

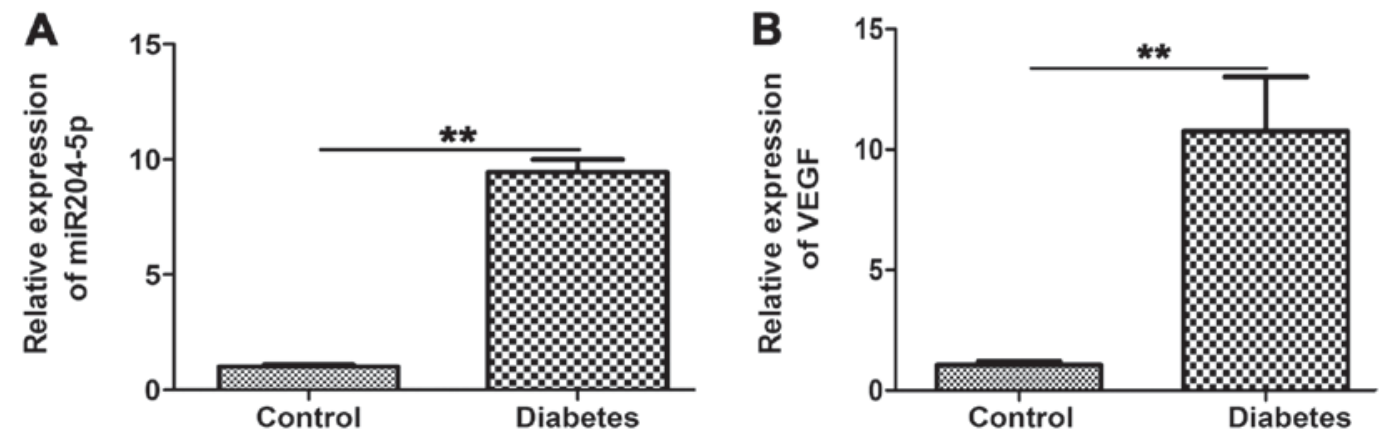

Figure 1. miR-204-5p and VEGF are overexpressed in STZ-induced diabetic rats. RNA was isolated from retina tissues of diabetic $(40 \mathrm{mg} / \mathrm{kg} \mathrm{STZ} \mathrm{treat-}$ ment) and control rats for analysis. The relative expression of (A) miR-204-5p and (B) VEGF was normalized to U6 and $\beta$-actin, respectively. ${ }^{* *} \mathrm{P}<0.01$. miR, microRNA; VEGF, vascular endothelial growth factor; STZ, streptozotocin.

A
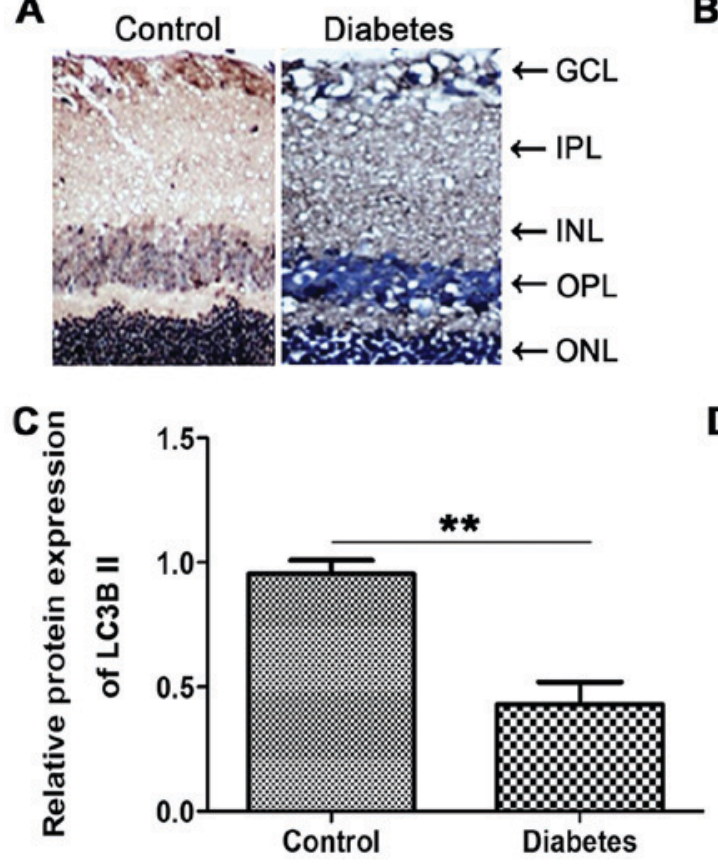

B

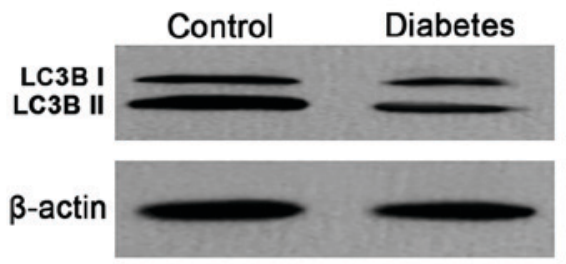

D

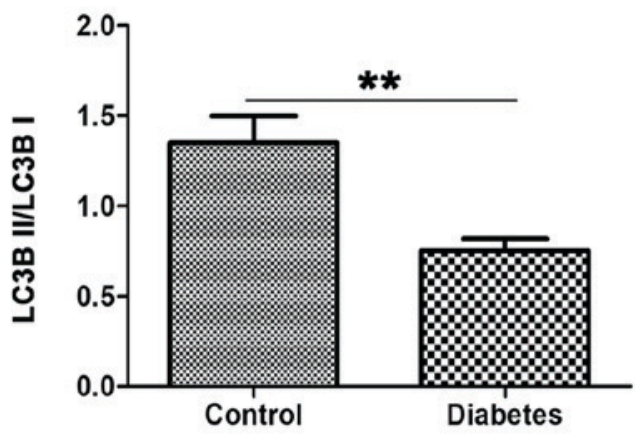

Figure 2. LC3B expression is inhibited in diabetic rats. Rats of the diabetes group were injected with streptozotocin (40 mg/kg) to induce diabetes. (A) Retinal tissues were extracted for immunohistochemistry and LC3B was detected by brown or dark yellow staining. (B) Western blot images and quantitative analysis of (C) LC3B-II protein expression and (D) the LC3B-II/LC3B-I ratio. ${ }^{* * *} \mathrm{P}<0.01$. GCL, ganglion cell layer; IPL, inner plexiform layer; INL, inner nuclear layer; LC3B, microtubule-associated protein 1 light chain 3; OPL, outer plexiform layer; ONL, outer nuclear layer.

significantly upregulated miR-204-5p expression $(\mathrm{P}<0.01)$ The results suggested that anti-miR-204-5p and miR-204-5p mimic were functional in diabetic rats. In addition, compared with the neg-miR group, injection of anti-miR-204-5p into diabetic rats significantly enhanced LC3B-II expression and the ratio of LC3B-II/LC3B-I (P<0.01; Fig. 3B and C). In the presence of the miR-204-5p mimic these levels decreased compared with the neg-miR control $(\mathrm{P}<0.01)$. mRNA expression levels of VEGF were significantly upregulated in the anti-miR-204-5p and the mimic groups compared with the neg-miR control $(\mathrm{P}<0.01$; Fig. 3D). Interestingly, miR-204-5p mimic treatment induced a larger increase in VEGF mRNA expression levels compared with the anti-miR-204-5p group.

In order to confirm the results in vitro, rRECs were isolated from diabetic rats and transfected with anti-miR-204-5p, miR-204-5p mimic or neg-miR. The purity of rRECs following isolation was determined via factor VII staining.
The percentage of factor VII-positive cells was $>97 \%$, indicating that rRECs were successfully isolated from retina tissues of diabetic rats (Fig. 4A). As presented in Fig. 4B, compared with the neg-miR group, anti-miR-204-5p markedly increased the number of autophagic vacuoles and a reduction was observed in response to treatment with miR-204-5p mimic. The results of the transfection efficiency analysis are presented in Fig. 4C; it was determined that anti-miR-204-5p successfully inhibited and miR-204-5p mimic successfully enhanced miR-204-5p expression compared with the neg-miR control $(\mathrm{P}<0.01)$. Anti-miR-204-5p further significantly promoted LC3B-II expression and significantly increased the LC3B-II/LC3B-I ratio; miR-204-5p mimic exhibited opposing effects compared with the neg-miR control (all $\mathrm{P}<0.01$; Fig. 4D and E). Additionally, anti-miR-204-5p and miR-204-5p mimic significantly upregulated the expression of VEGF compared with the neg-miR control $(\mathrm{P}<0.01$; Fig. 4F). 

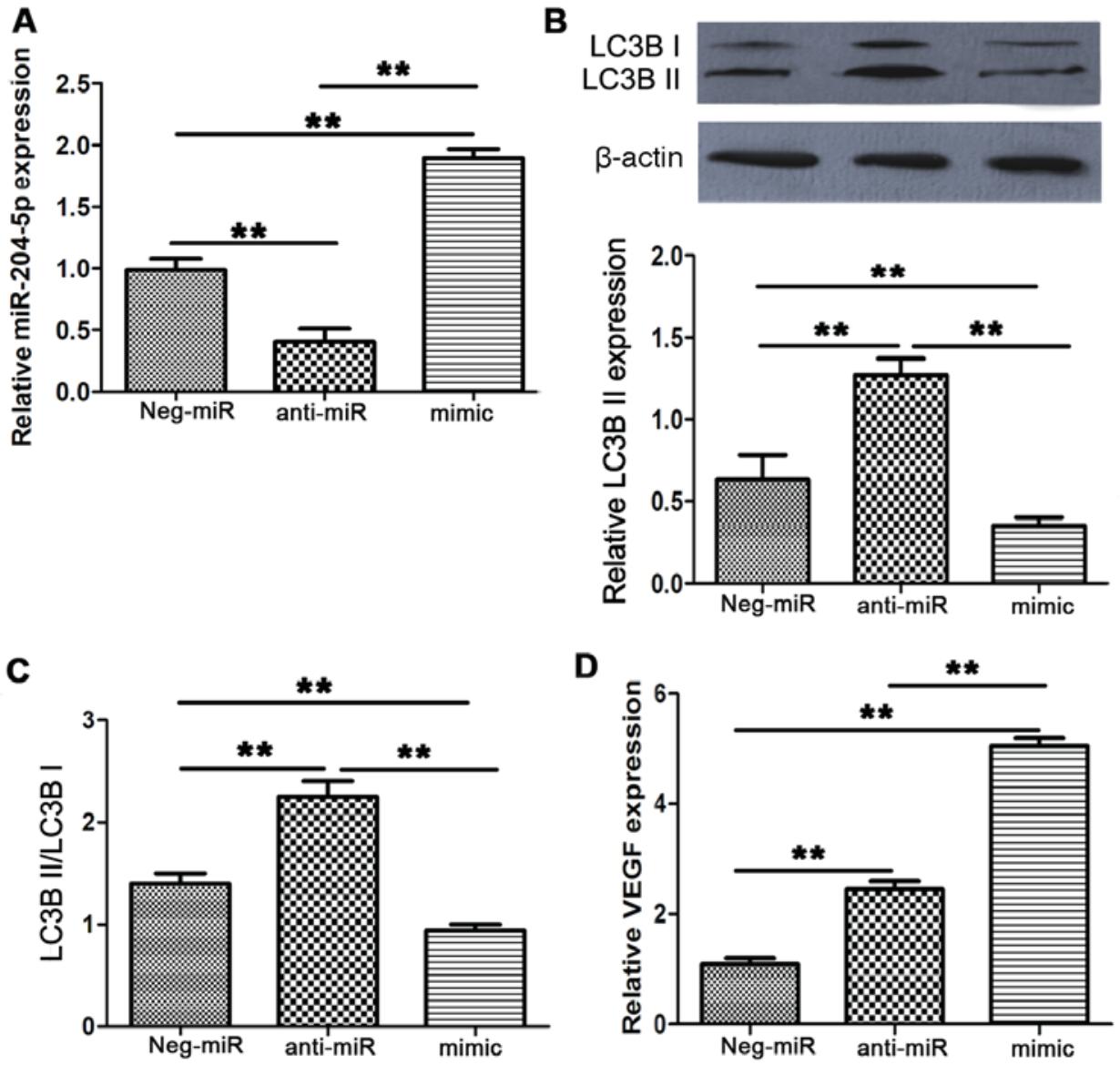

Figure 3. miR-204-5p downregulates the expression of LC3B-II and the ratio of LC3B-II/LC3B-I in vivo. Diabetic rats were treated with equal amounts of anti-miR, mimic and neg-miR and retina tissues were isolated for analysis. (A) Expression levels of miR-204-5p in isolated retina tissues. (B) Western blot images and quantitative analysis of (C) LC3B-II protein expression and (D) the LC3B-II/LC3B-I ratio. (D) mRNA expression levels of VEGF. Relative expression of miR-204-5p was normalized to U6 and VEGF was normalized to $\beta$-actin. ${ }^{* *} \mathrm{P}<0.01$. miR, microRNA; LC3B, microtubule-associated protein 1 light chain 3; VEGF, vascular endothelial growth factor; neg-miR, control miR; anti-miR, anti-miR-204-5p; mimic, miR-204-5p mimic.

These results revealed that miR-204-5p may be involved in the development of DR by negatively regulating the expression of LC3B-II.

\section{Discussion}

DR is a cause of blindness in individuals and affects their quality of life (23). It is critical to investigate the pathogenesis of DR and to explore novel treatment targets. In the present study, it was reported that miR-204-5p and VEGF mRNA were upregulated in the STZ-induced diabetes group and expression of LC3B-II and the LC3B-II/LC3B-I ratio were decreased in diabetic rats compared with the control. Furthermore, anti-miR-204-5p treatment promoted protein expression of LC3B-II and the LC3B-II/LC3B-I ratio compared with the neg-miR. Treatment with miR-204-5p mimic exhibited reverse effects compared with the control. These findings indicated that miR-204-5p may be involved in the progression of DR in retina tissues of diabetic rats by negatively regulating the expression of LC3B-II.

miRNAs are associated with various biological events, including cell proliferation, differentiation and apoptosis (24). Previous studies revealed that miR-204 suppresses the development of numerous types of cancer, including renal cell carcinoma (25), breast cancer (26) and gastric cancer (27). A recent study demonstrated that miR-204-5p exerts antitumor effects on melanoma (28) and inhibits the invasion and metastasis of laryngeal squamous cell carcinoma by suppressing forkhead box C1 (29). Furthermore, it was reported that miR-204-5p suppresses inflammation in renal tubular epithelial cells by directly targeting the interleukin-6 receptor (30). Few studies have investigated the function of miR-204-5p in the progression of DR; however, the upregulation of miR-204 in DR has been reported previously (19). In the STZ-induced diabetes model of the present study, the expression of miR-204-5p was upregulated in retina tissues compared with the control, which suggested a potential association between miR-204-5p and the development of DR. These findings indicated that miR-204-5p may be considered as a potential therapeutic target for DR treatment.

Autophagy is a conserved lysosomal degradation pathway that maintains cell homeostasis (31) and serves an adaptive role to protect organisms against infections and diabetic complications (32). Autophagy has been proposed as an important pathway associated with DR; thus, this process may serve as a potential therapeutic target for the treatment of DR (33). Expression of LC3B is a reported hallmark of the onset of autophagy (34) and the conversion of LC3B-I to LC3B-II is a common indicator of autophagy (35). LC3B-II is a specific marker of the autophagic process as its expression directly 


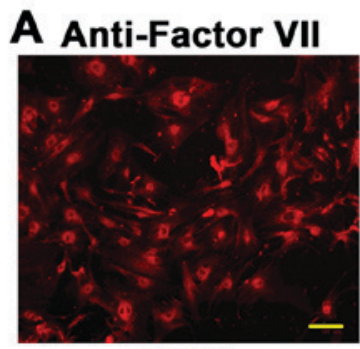

DAPI

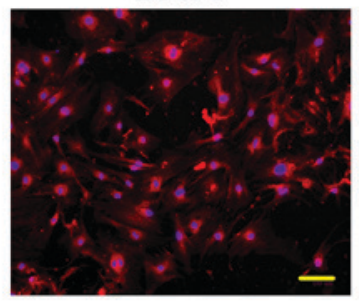

C

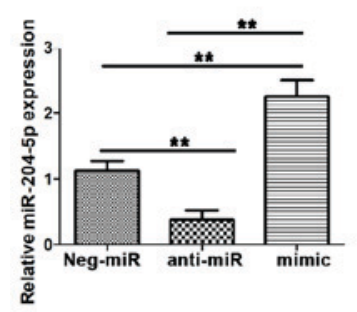

B Neg-miR
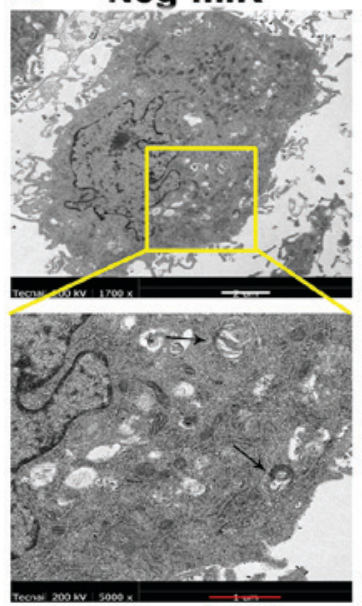

\section{D}
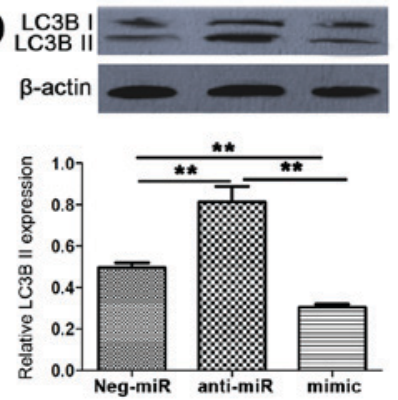

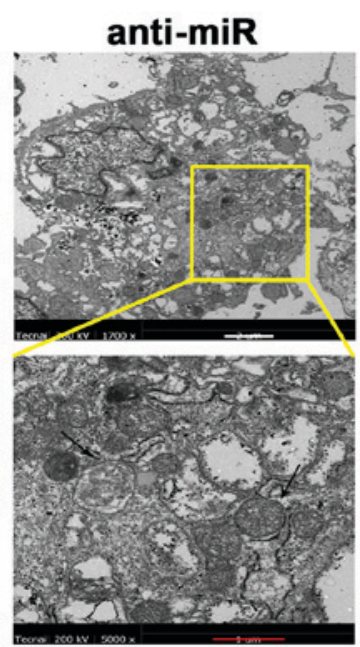

E

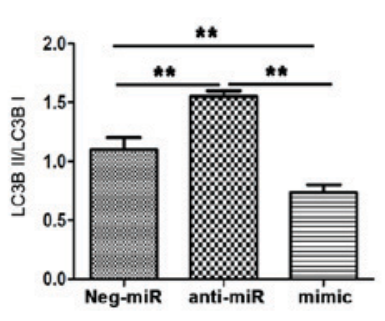

mimic
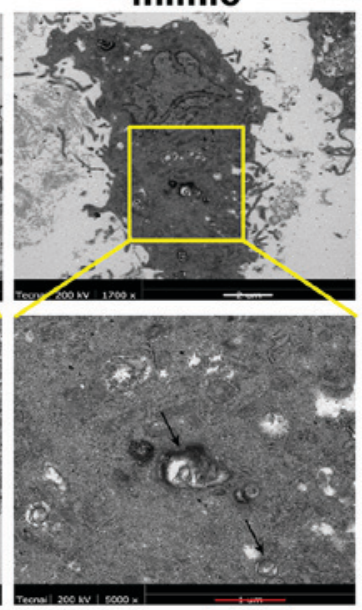

$\mathbf{F}$

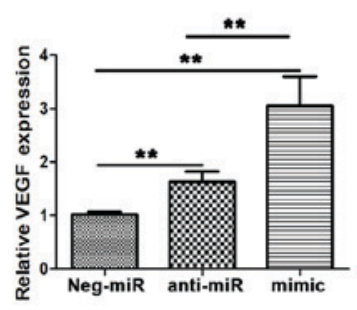

Figure 4. miR-204-5p downregulates the expression of LC3B-II and the ratio of LC3B-II/LC3B-I in vitro. rRECs were isolated from diabetic rats and transfected with anti-miR, mimic ant neg-miR. (A) Purity of rRECs was determined via factor VII staining; factor VII-positive cells are presented in red and nuclei in blue. Scale bar, $100 \mu \mathrm{m}$. (B) The amount of autophagic vacuoles was determined by transmission electron microscopy (magnification, $\mathrm{x} 1,700$ and $\mathrm{x} 5,000$ ). Scale bar in white, $2 \mu \mathrm{m}$; scale bar in red, $1 \mu \mathrm{m}$. (C) Expression levels of miR-204-5p in transfected cells. (D) Western blot images and quantitative analysis of LC3B-II protein expression and (E) the LC3B-II/LC3B-I ratio. (F) mRNA expression levels of VEGF. The relative expression of miR-204-5p was normalized to U6 and VEGF was normalized to $\beta$-actin. ${ }^{* *} \mathrm{P}<0.01$. rRECs, rat retinal endothelial cells; miR, microRNA; LC3B, microtubule-associated protein 1 light chain 3; VEGF, vascular endothelial growth factor; neg-miR, control miR; anti-miR, anti-miR-204-5p; mimic, miR-204-5p mimic.

correlates with the number of autophagosomes (11); however, the association between autophagy and DR is complex. Studies have demonstrated that STZ-induced diabetic rats exhibit increased levels of autophagy markers $(35,36)$. Conversely, it was reported that the number of autophagosomes was significantly decreased in diabetic rats and that high levels of glucose suppressed the ratio of LC3B-II/LC3B-I compared with that in the healthy control group (37). In the present study, it was observed that expression of LC3B-II and the LC3B-II/LC3B-I ratio were reduced in retina tissues of diabetic rats compared with the control group, suggesting a potential association between DR and autophagic activity. Additionally, anti-miR-204-5p promoted the expression of LC3B-II and the LC3B-II/LC3B-I ratio, which were suppressed in response to miR-204-5p mimic treatment compared with the neg-miR control. The data of the present study indicated that miR-204-5p negatively regulated LC3B-II expression in DR. Defective autophagy has been reported as an early event occurring in the pathogenesis of DR (10). These findings indicated a potential novel association between miR-204-5p, DR and autophagy.

VEGF induces the formation of new blood vessels and increases the permeability of existing vessels (38). VEGF is mainly expressed in ganglion cells and retinal pigment epithelial cells (39). Studies have revealed that VEGF expression is increased (40) and is a key factor in DR (41). In the present study, it was reported that the mRNA expression levels of VEGF in the diabetes group were significantly upregulated compared with the control group, which was in accordance with the findings of previous studies $(38,41)$. Anti-miR-204-5p and miR-204-5p mimic treatment promoted the expression of VEGF compared with the neg-miR control. These results revealed that miR-205-5p regulated the expression of VEGF in an unknown way.

The current study has several limitations. It was suggested that miR-204-5p may be associated with DR and autophagy by regulating the expression of the autophagy-specific marker LC3B-II; however, whether miR-204-5p directly or indirectly targets the expression of LC3B-II remains unknown. Thus, further research into the targets of miR-204-5p is required.

In conclusion, miR-204-5p and VEGF were reported to be upregulated in the retina tissues of diabetic rats and may be associated with the progression of DR. However, the mechanism by which miR-204-5p affected the VEGF levels remains unknown. Protein expression levels of LC3B-II and the LC3B-II/LC3B-I ratio were reduced in retina tissues of diabetic rats compared with the control. In diabetic rats and isolated rRECs, miR-204-5p-mimic treatment downregulated LC3B-II expression compared with the neg-miR control. These results revealed that miR-204-5p promoted the development 
of DR via downregulating the expression of LC3B-II. These results may further provide novel insight into the association between DR and autophagy.

\section{Acknowledgements}

Not applicable.

\section{Funding}

The current study was supported by Jiangxi Natural Science Foundation of China (grant no. 20151BAB205096).

\section{Availability of data and materials}

All data generated or analyzed during this study are included in this published article.

\section{Authors' contributions}

XBM performed PCR and western blot analyses, analyzed the data and prepared the original manuscript. YHC established the rRECs, performed the TEM analysis and contributed to preparing the figures. YYX designed the study and revised the manuscript. All authors read and approved the final version of the manuscript.

\section{Ethics approval and consent to participate}

The present study was approved by the Animal Ethics Committee of Second Affiliated Hospital of Nanchang University (Nanchang, China).

\section{Patient consent for publication}

Not applicable.

\section{Competing interests}

The authors declare that they have no competing interests.

\section{References}

1. Santos LL, Lima FJC, Sousa-Rodrigues CF and Barbosa FT: Use of SGLT-2 inhibitors in the treatment of type 2 diabetes mellitus. Rev Assoc Med Bras (1992) 63: 636-641, 2017.

2. Murchison AP, Hark L, Pizzi LT, Dai Y, Mayro EL, Storey PP, Leiby BE and Haller JA: Non-adherence to eye care in people with diabetes. BMJ Open Diabetes Res Care 5: e000333, 2017.

3. Leasher JL, Bourne RR, Flaxman SR, Jonas JB, Keeffe J, Naidoo N, Pesudovs K, Price H, White RA, Wong TY, et al: Erratum. Global estimates on the number of people blind or visually impaired by diabetic retinopathy: A meta-analysis from 1990-2010. Diabetes Care 2016;39:1643-1649. Diabetes Care 39: 2096, 2016

4. Arroba AI, Campos-Caro A, Aguilar-Diosdado M and Valverde AM: IGF-1, inflammation and retinal degeneration: A close network. Front Aging Neurosci 10: 203, 2018.

5. Hou L, Wei L, Zhu S, Wang J, Quan R, Li Z and Liu J: Avian metapneumovirus subgroup $C$ induces autophagy through the ATF6 UPR pathway. Autophagy 13: 1709-1721, 2017.

6. Wang L, Feng D, Liu Y, Li S, Jiang L, Long Z and Wu Y: Autophagy plays a protective role in motor neuron degeneration following spinal cord ischemia/reperfusion-induced spastic paralysis. Am J Transl Res 9: 4261-4270, 2017.
7. Gao N, Wang H, Yin H and Yang Z: Angiotensin II induces calcium-mediated autophagy in podocytes through enhancing reactive oxygen species levels. Chem Biol Interact 277: 110-118, 2017.

8. Wang T, Zhang L, Hu J, Duan Y, Zhang M, Lin J, Man W, Pan X, Jiang Z, Zhang G, et al: Mst1 participates in the atherosclerosis progression through macrophage autophagy inhibition and macrophage apoptosis enhancement. J Mol Cell Cardiol 98: 108-116, 2016.

9. Dehdashtian E, Mehrzadi S, Yousefi B, Hosseinzadeh A, Reiter RJ, Safa M, Ghaznavi H and Naseripour M: Diabetic retinopathy pathogenesis and the ameliorating effects of melatonin; involvement of autophagy, inflammation and oxidative stress. Life Sci 193: 20-33, 2018.

10. Lopes de Faria JM, Duarte DA, Montemurro C, Papadimitriou A, Consonni SR and Lopes de Faria JB: Defective autophagy in diabetic retinopathy. Invest Ophthalmol Vis Sci 57: 4356-4366, 2016.

11. Kabeya Y, Mizushima N, Ueno T, Yamamoto A, Kirisako T, Noda T, Kominami E, Ohsumi Y and Yoshimori T: LC3, a mammalian homologue of yeast Apg8p, is localized in autophagosome membranes after processing. EMBO J 19: 5720-5728, 2000.

12. Alizadeh S, Mazloom H, Sadeghi A, Emamgholipour S, Golestani A, Noorbakhsh F, Khoshniatnikoo M and Meshkani R: Evidence for the link between defective autophagy and inflammation in peripheral blood mononuclear cells of type 2 diabetic patients. J Physiol Biochem 74: 369-379, 2018.

13. Chandra S, Vimal D, Sharma D, Rai V, Gupta SC and Chowdhuri DK: Role of miRNAs in development and disease: Lessons learnt from small organisms. Life Sci 185: 8-14, 2017.

14. Nadeem A, Ashraf MR, Javed M, Hussain T, Tariq MS and Babar ME: Review-microRNAs: A new paradigm towards mechanistic insight of diseases. Pak J Pharm Sci 31: 2017-2026, 2018.

15. Gong Q, Xie J, Liu Y, Li Y and Su G: Differentially expressed micrornas in the development of early diabetic retinopathy. J Diabetes Res 2017: 4727942, 2017.

16. Chen Q, Qiu F, Zhou K, Matlock HG, Takahashi Y, Rajala RVS, Yang Y, Moran E and Ma JX: Pathogenic role of microRNA-21 in diabetic retinopathy through downregulation of PPAR $\alpha$. Diabetes 66: 1671-1682,2017.

17. Haque R, Iuvone PM, He L, Choi KSC, Ngo A, Gokhale S, Aseem M and Park D: The MicroRNA-21 signaling pathway is involved in prorenin receptor (PRR)-induced VEGF expression in ARPE-19 cells under a hyperglycemic condition. Mol Vis 23: 251-262, 2017.

18. Gomaa AR, Elsayed ET and Moftah RF: MicroRNA-200b expression in the vitreous humor of patients with proliferative diabetic retinopathy. Ophthalmic Res 58: 168-175, 2017.

19. Wu JH, Gao Y, Ren AJ, Zhao SH, Zhong M, Peng YJ, Shen W, Jing M and Liu L: Altered microRNA expression profiles in retinas with diabetic retinopathy. Ophthalmic Res 47: 195-201, 2012.

20. Gao J, Wang Y, Zhao X, Chen P and Xie L: MicroRNA-204-5p-mediated regulation of SIRT1 contributes to the delay of epithelial cell cycle traversal in diabetic corneas. Invest Ophthalmol Vis Sci 56: 1493-1504, 2015.

21. Nathan DM, Buse JB, Davidson MB, Heine RJ, Holman RR, Sherwin R and Zinman B: Management of hyperglycaemia in type 2 diabetes: A consensus algorithm for the initiation and adjustment of therapy. A consensus statement from the American diabetes association and the european association for the study of diabetes. Diab Tologia 49: 1711-1721, 2006.

22. Livak KJ and Schmittgen TD. Analysis of relative gene expression data using real-time quantitative PCR and the 2(-Delta Delta C(T)) method. Methods 25: 402-408, 2001.

23. Rubsam A, Parikh S and Fort PE: Role of Inflammation in diabetic retinopathy. Int J Mol Sci 19: pii: E942, 2018

24. Kaneko $\mathrm{H}$ and Terasaki H: Biological involvement of MicroRNAs in proliferative vitreoretinopathy. Transl Vis Sci Technol 6: 5, 2017.

25. Wu D, Pan H, Zhou Y, Zhang Z, Qu P, Zhou J and Wang W: Upregulation of microRNA-204 inhibits cell proliferation, migration and invasion in human renal cell carcinoma cells by downregulating SOX4. Mol Med Rep 12: 7059-7064, 2015.

26. Liu J and Li Y: Trichostatin A and Tamoxifen inhibit breast cancer cell growth by miR-204 and ERalpha reducing AKT/mTOR pathway. Biochem Biophys Res Commun 467: 242-247, 2015.

27. Lorenzon L, Cippitelli C, Avantifiori R, Uccini S, French D, Torrisi MR, Ranieri D, Mercantini P, Canu V, Blandino G and Cavallini M: Down-regulated miRs specifically correlate with non-cardial gastric cancers and Lauren's classification system. J Surg Oncol 116: 184-194, 2017. 
28. Palkina N, Komina A, Aksenenko M, Moshev A, Savchenko A and Ruksha T: miR-204-5p and miR-3065-5p exert antitumor effects on melanoma cells. Oncol Lett 15: 8269-8280, 2018.

29. Gao W, Wu Y, He X, Zhang C, Zhu M, Chen B, Liu Q, Qu X, Li W, Wen S and Wang B: MicroRNA-204-5p inhibits invasion and metastasis of laryngeal squamous cell carcinoma by suppressing forkhead box C1. J Cancer 8: 2356-2368, 2017.

30. Li H, Wang J, Liu X and Cheng Q: MicroRNA-204-5p suppresses IL6-mediated inflammatory response and chemokine generation in HK-2 renal tubular epithelial cells by targeting IL6R. Biochem Cell Biol: 2018 (Epub ahead of print).

31. Mizushima N, Levine B, Cuervo AM and Klionsky DJ: Autophagy fights disease through cellular self-digestion. Nature 451: 1069-1075, 2008

32. Volpe CMO, Villar-Delfino PH, Dos Anjos PMF and Nogueira-Machado JA: Cellular death, reactive oxygen species (ROS) and diabetic complications. Cell Death Dis 9: 119, 2018.

33. Levine B and Kroemer G: Autophagy in the pathogenesis of disease. Cell 132: 27-42, 2008.

34. Yoshii SR and Mizushima N: Monitoring and measuring autophagy. Int J Mol Sci 18: pii: E1865, 2017

35. Li XC, Hu QK, Chen L, Liu SY, Su S, Tao H, Zhang LN, Sun T and He LJ: HSPB8 promotes the fusion of autophagosome and lysosome during autophagy in diabetic neurons. Int J Med Sci 14: $1335-1341,2017$.

36. Cai X, Li J, Wang M, She M, Tang Y, Li H and Hui H: GLP-1 treatment improves diabetic retinopathy by alleviating autophagy through GLP-1R-ERK1/2-HDAC6 signaling pathway. Int J Med Sci 14: 1203-1212, 2017.

37. Kobayashi S, Xu X, Chen K and Liang Q: Suppression of autophagy is protective in high glucose-induced cardiomyocyte injury. Autophagy 8: 577-592, 2012.
38. Rodrigues M, Xin X, Jee K, Babapoor-Farrokhran S, Kashiwabuchi F, Ma T, Bhutto I, Hassan SJ, Daoud Y, Baranano D, et al: VEGF secreted by hypoxic muller cells induces MMP-2 expression and activity in endothelial cells to promote retinal neovascularization in proliferative diabetic retinopathy. Diabetes 62: 3863-3873, 2013.

39. Nishijima K, Ng YS, Zhong L, Bradley J, Schubert W, Jo N, Akita J, Samuelsson SJ, Robinson GS, Adamis AP and Shima DT: Vascular endothelial growth factor-A is a survival factor for retinal neurons and a critical neuroprotectant during the adaptive response to ischemic injury. Am J Pathol 171: 53-67, 2007.

40. Gilbert RE, Vranes D, Berka JL, Kelly DJ, Cox A, Wu LL, Stacker SA and Cooper ME: Vascular endothelial growth factor and its receptors in control and diabetic rat eyes. Lab Invest 78: 1017-1027, 1998

41. Bai Y, Ma JX, Guo J, Wang J, Zhu M, Chen Y and Le YZ: Muller cell-derived VEGF is a significant contributor to retinal neovascularization. J Pathol 219: 446-454, 2009.

This work is licensed under a Creative Commons Attribution-NonCommercial-NoDerivatives 4.0 International (CC BY-NC-ND 4.0) License. 